\title{
Anatomia, diagnóstico e tratamento de anquiloglossia na primeira infância
}

\author{
Anatomy, diagnosis and treatment of ankyloglossia in early childhood
}

Anatomía, diagnóstico y tratamiento de la anquiloglosia en la primera infancia

Josciane Dandara Lopes Gomes ${ }^{1 *}$, Rosimeire Cavalcante de Freitas ${ }^{1}$, Thaiana Nunes da Costa ${ }^{1}$, Aline Maquiné Pascareli Carlos ${ }^{1}$.

\begin{abstract}
RESUMO
Objetivo: Abordar através de uma revisão narrativa sobre anatomia, diagnóstico e tratamento de anquiloglossia na 10 infância para mostrar a relevância de uma intervenção odontológica nesses casos. Revisão Bibliográfica: A prega mucosa que vai da metade da face inferior da língua até o assoalho da boca é denominada de frênulo de língua. A palavra "anquiloglossia" surge das palavras gregas "agkilos" (curvas) e "glossa" (língua). Trata-se de uma condição anatômica em que o sujeito nasce com um frênulo curto, que no caso pode ou não impactar na vida do indivíduo, pois limita movimentos que podem ocasionar mudanças na fala. A relevância de uma intervenção odontológica para com os casos de anquiloglossia pode favorecer um melhor desempenho da língua, não prejudicando as inúmeras funções desta estrutura. No desenvolvimento do tratamento, é fundamental o procedimento cirúrgico, com o objetivo uma melhor flexibilidade lingual, aliado ao tratamento fonoaudiológico objetivando o restabelecimento da fonação e fisiologia normal de deglutição. Considerações Finais: Quando o problema é bem assinalado poderá cooperar para o melhor progresso do psíquico-emocional do indíviduo, proporcionando uma melhor mobilidade do movimento da língua, assim como uma melhor pronúncia das palavras.
\end{abstract}

Palavras-chave: Freio lingual em bebês, Frênulo lingual, Teste da linguinha, Anquiloglossia, Frenotomia.

\begin{abstract}
Objective: Approach through a narrative review on anatomy, diagnosis and treatment of ankyloglossia in early childhood to show the relevance of a dental intervention in those cases. Bibliographic Review: The mucous fold that runs from the lower half of the tongue to the floor of the mouth is called the tongue frenulum. The word "ankyloglossia" comes from the Greek words "agkilos" (curves) and "glossa" (tongue). It's an anatomical condition in which the subject is born with a short frenulum, which may or may not impact the individual's life, as it limits movements that can result in changes in the speech. The relevance of a dental intervention for cases of ankyloglossia may favor a better performance of the tongue, without harming the functions of this structure. In the development of the treatment, the surgical procedure is fundamental with the objective of a better lingual flexibility allied to the speech therapy, aiming at the reestablishment of phonation and normal swallowing physiology. Final Considerations: When the problem is well marked, it can cooperate for the better progress of the individual's psychic-emotional, providing better mobility of the movement of the tongue, as well as a better pronunciation of the words.
\end{abstract}

Keywords: Lingual brake in babies, Lingual frenulum, Tongue screening test, Ankyloglossia, Phrenotomy.

\section{RESUMEN}

Objetivo: Abordaje a través de una revisión narrativa sobre anatomía, diagnóstico y tratamiento de la anquiloglosia en la primera infancia para mostrar la relevancia de una intervención dental en estos casos. Revisión Bibliográfica: El pliegue mucoso que va desde la mitad inferior de la lengua hasta el piso de la boca se llama frenillo lingual. La palabra "anquiloglosia" surge de las palabras griegas "agkilos" (curvas) y "glossa" (lengua). Es una condición anatómica en la que el sujeto nace con un frenillo corto, que en este caso puede afectar o no la vida del individuo, ya que limita los movimientos que pueden provocar cambios en el habla. La relevancia de una intervención dental para casos de anquiloglosia puede favorecer un mejor desempeño de la lengua sin perjudicar las funciones de esta estructura. En el desarrollo del tratamiento, el procedimiento quirúrgico es fundamental, con el objetivo de una mejor flexibilidad lingual, aliado al tratamiento logopédico que tiene como objetivo el restablecimiento de la fonación y la fisiología normal de la deglución. Consideraciones Finales: Cuando el problema está bien marcado, se puede cooperar para mejor progreso psíquico-emocional del individuo, proporcionando mejor movilidad del movimiento de la lengua y pronunciación de las palabras.

Palabras clave: Freno lingual en bebés, Frenillo lingual, Test de la lengüita, Anquiloglosia, Frenotomía.

${ }^{1}$ Centro Universitário do Norte (Uninorte), Manaus - AM. *E-mail: joscianedandara@gmail.com 


\section{INTRODUÇÃO}

Os freios bucais são estruturas anatômicas essenciais para a mastigação, deglutição, fala e respiração. $O$ encurtamento dos freios limita os movimentos dos lábios e da língua. O freio lingual tem como função dificultar os movimentos da língua, interferindo na amamentação do bebê (PEIXOTO APM, et al., 2019). O frênulo lingual é a prega mucosa que começa de uma área mais fixa para uma parte com maior liberdade de movimentos. A palavra "anquiloglossia" surge das palavras gregas "agkilos" (curvas) e "glossa" (língua). Trata-se de uma condição anatômica em que o sujeito nasce com um frênulo curto, podendo ou não impactar na vida do indivíduo (ISAC C, 2018),

Os bebês avaliados e diagnosticados com frênulo lingual alterado são comumente submetidos a um procedimento cirúrgico, conhecido como frenotomia. A frenotomia pode ser parcial (frenulotomia) ou total (frenectomia) (FUJINAGA Cl, et al., 2016). Segundo Bistaffa AGI, et al. (2017), diante o que ao autor fala, existem diferentes técnicas para correção das inserções anormais do freio, entre elas está a frenotomia e a frenectomia. A cirurgia de frenotomia envolve a incisão da inserção no periósteo, já a frenectomia envolve a excisão completa do freio e de sua inserção no periósteo, sendo assim indicada no caso de freio grande e volumoso.

As principais alterações desses frênulo da língua podem ocasionar diversas sequelas como manutenção contínua da posição da boca entreaberta, alterações oclusais e periodontais, limitação nos movimentos linguais e postura baixa da língua na cavidade bucal (ALMEIDA KR, et al., 2017). Tais limitações podem causar comprometimento mastigatório, na deglutição e fala. Por isso, pode ser indicada a intervenção cirúrgica para corrigir as alterações do frênulo da língua, geralmente, sendo realizada por profissionais como cirurgiões dentistas e otorrinolaringologistas (SANTOS S e BARBOSA GF, 2017).

A língua tem como característica clínica de Anquiloglossia um freio anormalmente curto sendo ele em formato normal, porém há uma dificuldade quando se faz a elevação da língua sobre o palato, essa anomalia resulta na limitação dos movimentos da língua, interferindo também na fala e amamentação (FERREIRA LSR, et al., 2018).

O frênulo lingual é analisado como característica de normal ou alterado, dependendo do critério utilizado feito pelo profissional que está avaliando esse frênulo, dessa forma os diagnósticos mais utilizados são a partir da observação visual, analisando a mobilidade da língua através de um exame físico realizado (MARCHESAN IQ, 2010).

No atual panorama, os protocolos de diagnóstico foram propostos no início dos anos 90 com a Assessment Tool for Lingual Frenulum (traduzido para português como "avaliação do frênulo lingual") que diz respeito à inspeção visual que é conhecido como o protocolo de Kotlow, reconhecidamente no Brasil como o "Teste da Linguinha", no entanto não existe um padrão para diagnostico da anquiloglossia (QUEIROZ IQD, 2019).

O Teste da Linguinha pode ser executado por profissionais com formação dentro da área de saúde, sendo cirurgião-dentista, pediatra e odontologista profissionais capacitados para detectar anormalidades que possam estar presentes na boca do recém-nascido. Com a finalidade de desempenhar o diagnóstico, manifestações precoces de anquiloglossia em bebês e para uma melhor denominação do tratamento a ser feito, o teste torna-se imprescindível, para uma melhor qualidade de vida sendo ela emocional ou funcional para uma criança e sua mãe (OLIVEIRA MTL, et al., 2019).

Após a realização da cirurgia no bebê, recomenda-se que faça o aleitamento materno imediatamente após a cirurgia, pois a partir disso é possível melhorar os movimentos de sucção feito pelo bebê na hora de mamar, mantém a calma do paciente e se espera melhora imediata ou até mesmo nas primeiras 72 horas. Sendo assim, obtém-se uma grande importância nos movimentos para a extração do leite materno durante a amamentação (ALMEIDA KR, et al., 2017; BISTAFFA AGI, et al., 2017).

O presente trabalho tem como finalidade de analisar através de uma revisão narrativa, pesquisada em portais sobre a anatomia, diagnóstico e tratamento de anquiloglossia na $1^{\circ}$ infância. 


\section{REVISÃO BIBLIOGRÁFICA}

\section{Anatomia da língua}

A língua é um órgão muscular, revestida por um estojo mucoso, em sua face superior atrás apresenta um sulco em forma de $\mathrm{V}$ o qual se encontram as papilas degustativas. Em sua face inferior apresenta as veias raninas e o freio da língua o qual limita seus movimentos (JUNIOR WMP, 2019).

A língua vem de uma parede ventral da orofaringe, na região dos quatro primeiros arcos branquiais. É a partir da quarta semana de gestação que as duas saliências do ectomesênquima surgem no aspecto interno do primeiro arco branquial, dando origem as saliências linguais (SILVA VF, et al., 2019).

Considera-se a língua normal quando ela apresenta duas classes de músculos, que seriam os músculos esqueléticos e os próprios. O que determina a mobilidade e a variabilidade morfológica do órgão é o entrecruzamento dos feixes musculares. O estiloglosso, hioglosso, genioglosso, palatoglosso, faringoglosso e amigdaloglosso são conhecidos como músculos extrínsecos. O estiloglosso se desenvolve através de fibras tendinosas e musculares na face anterior da apófise estilóide, caminha-se obliquamente a diante e para baixo, refletindo o aspecto de arco de concavidade anterior pela base da língua (AGUIAR FTB, et al., 2017).

O entrecruzamento dos feixes musculares da língua, movidos para a diante e as fibras do hioglosso compõem a parte principal da musculatura lingual longitudinal. Introduzindo-se no limite lateral do corpo do osso hióide, o hioglosso, assim como em seu corno maior até perto do ápice, manda suas fibras para cima, fasciculadas, que vão se desviando em direção anterior e medial. Se entrecruzam com os fascículos transversais e com feixes do estiloglosso, assim as fibras do hioglosso chegam no dorso da língua, na qual terminam (CAMPIGNION P, 2019).

Ainda segundo o autor acima, o feixe dependente do músculo hioglosso tem de se levar em consideração o músculo condroglosso, que a começar pelo corno menor do hióide que dirige para cima e para diante, unindo-se com as fibras do hioglosso, chega quase até a linha média da língua. O genioglosso é considerado o músculo esquelético mais corpulento da língua, tem origem ao lado da linha média através de fibras, em grande maioria tendinosas, gerada na espinha mentoniana, acima da inserção dos músculos gênio-hioideus.

O frênulo lingual é a prega mucosa que começa de uma área mais fixa para uma parte com maior liberdade de movimentos, e é conhecida como frênulo. A prega mucosa que vai da metade da face inferior da língua até o assoalho da boca (ARAÚJO AB, et al., 2015).

No que trata-se do frênulo de língua, é fundamental que se diferencie os termos freio e frênulo. $O$ freio é um pequeno pedaço de tecido que liga duas estruturas, sendo uma dela móvel. Pode ser definido como uma prega de pele que limita o alcance de movimento de uma estrutura. Já o frênulo é utilizado para denominar pequenas pregas (MARTINELLI RLC, 2015).

\section{Anquiloglossia}

No ano de 2013, foi divulgado a publicação do Protocolo de Avaliação do Frênulo da Língua que iria ser realizado em Bebês, levando em consideração todos os aspectos da história clínica, verificação anatomofuncional e avaliação da sucção nutritiva e não nutritiva, sendo estabelecida a validade de conteúdo e, para posteriormente poder concluir todas as etapas de validação. Dessa forma, na Triagem Neonatal, o denominado "Teste da Linguinha" deverá ser realizado durante as 48 horas após o nascimento, desempenhando a avaliação anatomofuncional que é aplicada, permitindo diagnosticar os casos mais graves e indicar a frenotomia lingual ainda na maternidade (MARTINELLI RLC, et al. 2016).

Tendo em vista esses aspectos, faz-se menção, novamente, a palavra "anquiloglossia" que surge das palavras gregas "agkilos" (curvas) e "glossa" (língua); tratando-se de uma condição anatômica em que o sujeito nasce com um frênulo curto, na qual é identificado a restrição do movimento da língua, que no caso pode ou não impactar na vida do indivíduo, pois limita movimentos que podem ocasionar mudanças na fala e deglutição. Essa mudança é visível já no nascimento, acontece da ponta da língua até o rebordo alveolar lingual (ISAC C, 2018). 
É uma condição hereditária, ou seja, parentes próximos podem manifestá-la. É indicado que seja uma desordem autossômica dominante, sendo sua transmissão entre gerações realizada pelo meio do cromossoma X. Seu mecanismo etiopatogênico exato ainda é desconhecido (SANTOS S e BARBOSA GF, 2017).

A anquiloglossia apresenta-se regularmente como uma anomalia individualizada. Entretanto demonstrase como um dos aspectos de síndromes raras assim como a síndrome da fenda palatina e do Kindler, o orodigitofacial ou síndrome de Opitz (RODRIGUES AFA, 2019).

Vale salientar que ao analisar as pessoas com anquiloglossia, nota-se que não há nenhuma outra anomalia ou doenças congênitas. Verifica-se que o uso de cocaína pelas mães durante a gestação pode influenciar para o risco de anquiloglossia, os bebês de mães usuárias dessa substância apresentam três vezes mais probabilidades de possuir anquiloglossia do que os bebês de mães saudáveis (COTA ALS, et al., 2019).

A grande preocupação com um diagnóstico e tratamento da anquiloglossia é antigo, entretanto a descrição de uma avaliação mais objetiva e o uso de instrumentos para triagem e diagnóstico é mais recente, mais precisamente a partir da década de 90 (ARAÚJO T, et al., 2017).

A identificação de anquiloglossia e seus critérios vão variar de pesquisa para pesquisa, muitos autores consideram e utilizam características físicas da anatomia bucal dos bebês. Os critérios podem ser sinais de comprometimento funcional, ou seja, se eles têm a capacidade ou não de projetar a língua, como também a diminuição de sua movimentação (MARCIONE ESS, et al., 2016).

Portanto, nessa conjuntura, o efeito que a anquiloglossia tem sobre a amamentação é considerável, pois ela causa dor e trauma mamilar para as mães. Todavia, ainda não existe um teste totalmente validado, considerado padrão-ouro no que se refere ao diagnóstico de anquiloglossia em neonatos (POMPÉIA LE, et al., 2017).

A anquiloglossia é a anomalia congênita mais diagnosticada e negligenciadas da nova geração, essa anomalia se não tratada pode influenciar de diversas formas na vida do indivíduo, trazendo consequências irreversíveis graves em diversas fases da sua vida. O diagnóstico é fundamentado em dois critérios, na qual um vai avaliar a estrutura anatômica, onde é feita uma inspeção e a outra avaliará a elevação, extensão e a lateralidade da língua. Todavia, para que haja uma avaliação mais completa, com indicação cirurgia, é fundamental que haja uma equipe multidisciplinar, como pediatra, fonoaudiólogo, odontopediatra, além de um clínico geral (OLIVEIRA MTL, et al., 2019).

\section{Classificação da anquiloglossia}

É imprescindível a realização de exames de rotina do frênulo lingual, onde através dele será identificado anormalidades na inserção, possibilitando desta forma, medidas preventivas. Ao amamentar, se a mãe sentir dor, se tiver lesão nos mamilos e dificuldade na sucção, todos esses podem ser sinais relacionados a mudanças no frênulo (DUTRA MRP, et al., 2020).

Os aspectos da língua e do frênulo devem ser abordados no exame clínico, levando em consideração a forma, espessura, movimentos das funções, fixação. Se realizado de forma correta pode proporcionar um diagnóstico exato. Vale salientar que é fundamental que ele seja praticado usando protocolos específicos, para fundamentar um plano de tratamento sustentado com evidências clínicas (MARTINELLI RLC, 2015).

Colaborando com esse entendimento, Neto Júnior, et al. (2016) ressalta que no que se refere aos critérios anatômicos Kotlow, se tem determinada classificação da anquiloglossia, que foi baseada através da distância da inserção do freio lingual ao ápice da língua. A classificação é: Classe I (leve, 12-16mm), Classe II (moderada, 8-11 mm), Classe III (severa, 3-7mm) e Classe IV (completa, <3mm). Estas duas últimas precisam de mais atenção, porque restringem gravemente o movimento da língua (NETO JÚNIOR, et al., 2016).

Portanto, para classificar a anquiloglossia é necessário observar algumas características sobre a amplitude dos movimentos da língua, que no caso são estabelecidos pelos critérios de Kotlow. Assim, de acordo com os critérios, a ponta da língua deve ser apta a se projetar para fora da boca (sem fissuras), varrer tanto os lábios superiores, quanto os inferiores facilmente. E, quando retraída, a língua não deve causar isquemia ou força excessiva na região dos dentes anteriores (QUEIROZ IQD, 2019). 


\section{Diagnóstico e correção da anquiloglossia}

As diversas consequências da anquiloglossia na hora da amamentação são questionáveis na literatura, onde ocorre na minoria dos casos específicas. A dificuldade de amamentação ocorre quando a criança precisa se alimentar e não consegue fazer a sucção do mamilo com os lábios e a língua, o que impede o fechamento adequado, causando desconforto e dor dos mamilos, e dificuldade de sucção, assim o bebê tem perda de peso e acontece o desmame precoce (QUEIROZ IQD, 2019).

Para reduzir e evitar riscos de complicações, é indicado a correção da anquiloglossia logo na infância. A equipe multidisciplinar (pediatra, fonoaudiólogo, odontopediatra e o clinico geral) é que detectará as anormalidades na cavidade bucal de recém-nascidos. Porém, com o fortalecimento da odontologia para bebês, logo a correção de anquiloglossia e outras complicações tornou-se possível a partir o diagnóstico destas anomalias bucais (SANTOS HKMPS, 2019).

O fonoaudiólogo sobre os casos de anquiloglossia atua averiguando as reais e as condições do frênulo da língua com examinação visual, observando a mobilidade da língua e analisando as funcionalidades orofaciais de deglutição, mastigação e fala. Quando primordial, é necessária uma avaliação de um outro profissional, devendo fazer a devida intervenção cirúrgica ou fonoterapia para reparação das modificações localizadas (BRITO SF, et al., 2008).

Com a finalidade de unificar os critérios de diagnóstico entre os profissionais da saúde, a proposta de protocolo baseia-se nas diversas variações anatômicas do frênulo e suas interferências funcionais. É fundamental que a avaliação clínica seja breve e não invasiva, que seja menos estressante ao recém-nascido, proporcionando uma prática embasada em indícios para uma melhor conduta terapêutica (FRAGA MRBA, et al., 2020). Em suma, para os procedimentos com o freio lingual são dados por meio de um pequeno procedimento cirúrgico do tecido mole, praticado entre as aberturas dos ductos da glândula mandibular, a frenectomia pode ser feita de maneira similar, devendo ser desenvolvida sobre os ductos da glândula submandibular (COELHO MDRBA, et al., 2020).

O frênulo proporciona uma movimentação livre da língua. Dessa maneira, perante a evolução embrionária, quando não há o acontecimento do apoptose integral do frênulo, no qual o tecido residual pode prejudicar uma flexibilidade lingual e, em vista disso, as utilidades orais, sendo capaz de ocasionar à anquiloglossia (MARCIONE ESS, et al., 2016) O frênulo lingual é conceituado como uma minúscula membrana mucosa, no entanto existe uma diversidade de variação anatômica, sendo nomeada de inúmeras nomenclaturas: anquiloglossia parcial ou total, língua presa, freio curto, muscular (MARTINELLI RCA, 2015).

Martinelli RCA, et al. (2012) recomendaram um protocolo fundamentado em uma revisão de literatura, observando as diversas alterações anatômicas do frênulo lingual, tal como as funções de deglutição e sucção. A finalidade deste protocolo foi imenso progresso do diagnóstico da intervenção, do frênulo sobre os movimentos da língua durante a amamentação. Todavia, sua aplicação demonstrou uma longa e complexo, assinalando a indispensabilidade de adequações para se tornar um mecanismo fácil e rápido de aplicação (MARTINELLI RCA, 2013).

Quando identificado em bebês deve ser realizado o tratamento com a frenotomia lingual, onde pode haver uma melhora significativa durante a sucção e amamentação do recém-nascido, evidenciando assim uma relevante intervenção para melhoria de qualidade de vida, da mãe e da criança (SAMPAIO C, 2018). Dessa forma se não houver o tratamento adequado e ágil poderá acarretar no decorrer da vida social dos indivíduos alguns problemas graves, tal como ter dificuldade de utilizar instrumento de sopro, dificuldade de falar. $\mathrm{O}$ tratamento em pacientes de primeira infância é uma forma que visa prevenir as frustrações futuras, devido a limitação da língua que venham acarretar ao indivíduo (RIBEIRO FP, et al., 2016).

Uma vez identificada, existem dois tipos de tratamentos, o primeiro tratamento é o conservador quanto a não realizar uma intervenção cirúrgica, onde será trabalhado um tratamento aliado à fonoterapia, com o objetivo de alongamento da estrutura do freio lingual, e também o segundo, chamado de não conservador, sendo referente a intervenção cirúrgica (OLIVEIRA DAM, et al., 2019). 
Em relação ao diagnóstico da avaliação da função anatomofuncional por meio do programa de triagem neonatal, foi analisada a postura labial em repouso dos bebês, sendo também confirmada a tendência do posicionamento da língua durante o choro e o formato da ponta da língua, quando a ponta da língua estava alta durante o choro e a ação elevação, analisado todos esses itens pode-se chegar uma conclusão, sobre a necessidade da liberação do frênulo lingual (ARRUDA EMG, et al, 2019). O diagnóstico e o tratamento desse frênulo podem ser orientados por profissionais como os médicos, pediatras, odontopediatras e fonoaudiólogos. Porém, alguns desses profissionais não são devidamente treinados e não conseguem realizar o diagnóstico adequado e a correção cirúrgica correta dos pacientes com anquiloglossia (PINTO ABR, et al., 2019).

A técnica cirúrgica para o freio lingual é denominada de frenotomia, que consiste na incisão linear anteroposterior do freio lingual, sem retirada alguma de tecido (PROCÓPIO IMS, et al., 2017). A técnica mais usual para a soltura do frênulo lingual é a cirurgia de frenectomia (BISTAFFA AGI, et al., 2017).

O procedimento cirúrgico simplificado, seguro e eficiente, consistindo em realização de um pequeno corte no freio lingual da criança, devendo ser realizado por profissionais como otorrinolaringologistas, pediatras e médicos dentistas (XAVIER MMAPC, 2014). Após a intervenção cirúrgica o acompanhamento de um fonoaudiólogo é extremamente essencial para uma melhor avaliação sobre a movimentação da língua isolada e verificando o bom desempenho das funções orais (RODRIGUES AFA, 2015).

Os profissionais recomendados para os tratamentos com o frênulo lingual são odontopediatras, o pediatra e o clínico geral comprovadamente capacitados para realizar a identificação das anomalias na boca de recémnascidos e crianças (VIEIRA KA e MACHADO FG, 2018). No atual panorama com a inserção de especialidades de Odontologia para crianças tornou-se possível o diagnóstico de alterações bucais, como dente neonatal nos primeiros dias de vida, pré-natal e anquiloglossia. Entretanto, é cada vez mais comum nas clínicas fonoaudiólogas, em fase infantil, as queixas de alterações na articulação da fala e respectivamente dos seus sons emitidos, onde a ligação direta é com o frênulo lingual (SUZART DD e CARVALHO ARR, 2016).

\section{CONSIDERAÇÕES FINAIS}

É necessário o conhecimento anatômico da língua do bebê, desta forma, o diagnóstico precoce pode ser realizado e o tratamento mais indicado aplicado. O desenvolvimento do presente estudo possibilitou uma análise sobre a relevância da frenulotomia devendo ser executada sempre que a Anquiloglossia ocasionar algum dano sobre as funções estomatognáticas e vida social de uma criança. Portanto quando bem assinalado, poderá cooperar para o melhor progresso do psícoemocional do indivíduo, na medida que irá proporcionar uma melhor mobilidade do movimento da língua, assim como uma melhor pronúncia das palavras.

\section{REFERÊNCIAS}

1. AGUIAR FTB, et al. Relação sobre características anatômicas e a anquiloglossia. Jornada Odontológica dos Acadêmicos da Católica, 2017; 3(1): 1-4.

2. ALMEIDA KR, et al. Frenotomia lingual em recém-nascido, do diagnóstico à cirurgia: relato de caso. Revista CEFAC, 2018; 20(2): 258-262.

3. ARAÚJO AB, et al. Caracterização do frênulo lingual em bebês usuários de uma Unidade Básica de Saúde na cidade de Ipatinga-MG. ÚNICA Cadernos Acadêmicos, 2015; 1(1).

4. ARAÚJO T, et al. Anquiloglossia: Causas, consequências e tratamento. Jornada Odontológica dos Acadêmicos da Católica, 2017; 2(1): 1-6.

5. BISTAFFA AGI, et al. Frenotomia lingual em bebê. Revista Uningá Review, 2017; 29(2): 1-5.

6. BRITO SF, et al. Frênulo lingual: classificaçao e conduta segundo ótica fonoaudiológica, odontológica e otorrinolaringológica. Revista CEFAC, 2008; 10(3): 343-351.

7. CAMPIGNION P. Cadeias posteroanteriores e anteroposteriores: Cadeias musculares e articulares-método GDS. Summus Editorial, 2019.

8. COELHO JV, et al. Abordagem Cirúrgica De Frenectomia Lingual: Um Relato De Caso. In: XXI FAVE - Fórum acadêmico da faculdade Vértice, 2019. Disponível em: https://fave.univertix.net/wp-content/uploads/2019/11/A45 ABORDAGEM-CIR\%C3\%9ARGICA-DE-FRENECTOMIA-LINGUAL-UM-RELATO-DE-CASO.pdf. Acesso em: 05 ago. 2020 . 
9. COTA ALS, et al. Frenectomia para restituição fono-motricial da língua. Revista Eletrônica Acervo Saúde, 2019; 35: e1457-e1457.

10. DUTRA MRP, et al. Indicadores de qualidade de triagem auditiva e de avaliação do frênulo lingual neonatal. CoDAS, São Paulo, 32(3): e20180179, 2020.

11. FERREIRA LSR, et al. Anquiloglossia: revisão de literatura. Caderno de Graduação-Ciências Biológicas e da Saúde UNIT-PERNAMBUCO, 2018; 3(3): 93.

12. FRAGA MRBA, et al. Anquiloglossia versus amamentação: qual a evidência de associação?. Revista CEFAC, 22(3): e12219, 2020.

13. FUJINAGA Cl, et al. Frênulo lingual e aleitamento materno: estudo descritivo. Audiology- Communication Research, 22: e1762, 2017.

14. GOMES E, et al. Freio lingual: abordagem clínica interdisciplinar da Fonoaudiologia e Odontopediatria. Revista da Associação Paulista de Cirurgiões Dentistas, 2015; 69(1): 20-24.

15. ISAC C. Frenectomia-momento ideal da intervenção cirúrgica. Tese de Doutorado (Doutorado em Medicina Dentária). Instituto Universitário Egas Moniz, Almada, 2018; 71p.

16. JUNIOR WMP, et al. Frenectomia na Primeira Infância. Revista Eletrônica Saúde Multidisciplinar da Faculdade Morgana Potrich, 2019; 6(1): 1-7.

17. MARCHESAN IQ. Protocolo De Avaliação Do Frênulo Da Língua. Speech, Language, Hearing Sciences and Education Journal, 2010;12(6): 977-989.

18. MARCIONE ESS, et al. Classificação anatômica do frênulo lingual de bebês. Revista CEFAC, 2016; 18(5): $1042-1049$.

19. MARTINELLI RLC, et al. Validade e confiabilidade da triagem: "teste da linguinha". Revista CEFAC - Speech, Language, Hearing Sciences and Education Journal, 2016; 18(6): 1323-1331.

20. MARTINELLI RLC. Validação do protocolo de avaliação do frênulo da língua em bebês. Tese de Doutorado. Universidade de São Paulo, São Paulo, 2015; 116p.

21. MARTINELLI RLC, et al. Protocolo de avaliação do frênulo lingual para bebês: relação entre aspectos anatômicos e funcionais. Revista CEFAC, 2013; 15(3): 599-610.

22. MOREIRA TFNS. Conhecimentos, atitudes e práticas dos médicos pediatras em relação à saúde oral. Tese de Dourados (Doutorado em Medicina Dentária). Universidade Católica Portuguesa, 2018; 117p.

23. OLIVEIRA DAM, et al. Frenectomia Lingual: Relato De Caso. Unifunec Ciências da Saúde e Biológicas, 2019; 3(5): 18.

24. OLIVEIRA MTP, et al. Frenotomia lingual em bebês diagnosticados com anquiloglossia pelo Teste da Linguinha: série de casos clínicos. Revista da Faculdade de Odontologia-UPF, 2019; 24(1): 73-81.

25. PEIXOTO APM, et al. Frenectomia lingual e labial superior em odontopediatria. Trabalho de Conclusão de Curso (Odontologia). Univale, Governador Valadares, 2019; $11 \mathrm{p}$.

26. POMPÉIA LE, et al. A influência da anquiloglossia no crescimento e desenvolvimento do sistema estomatognático. Revista Paulista de Pediatria, 2017; 35(2): 216-221.

27. PROCOPIO IMS, et al. Frenotomia lingual em lactentes. Revista da Faculdade de Odontologia-UPF, 2017; 22(1): 114119.

28. QUEIROZ IQD. Comparação entre dois protocolos para diagnóstico de Anquiloglossia em bebês nascidos no Hospital Universitário de Brasília. Dissertação (Mestrado em Odontologia). Universidade de Brasília, Brasília, 2019; 80p.

29. RIBEIRO FP, et al. Frenectomia lingual em paciente pediátrico: relato de caso. Revista da AcBO, 2016. 5(1): 23167262.

30. ROCHA MCP, et al. O Impacto da anquiloglossia na primeira infância e a importância da identificação precoce. Trabalho de Conclusão de Curso (Especialista em especialista em Atendimento Interdisciplinar Preventivo na Primeira Infância). Universidade Estadual de Campinas, Campinas, 2018; 25p.

31. RODRIGUES AFA. Fenótipo Crânio-Oro-Facial em Situações de Alterações Dentárias de Número. Monografia (Bacharel em Medicina Dentária). Universidade do Porto, Porto, 2019; 64p.

32. SAMPAIO C. Frenotomia lingual em paciente pediátrico: relato de caso. Revista Visão Universitária, 2018. 2(1).

33. SANTOS HKMPS. Efeito da frenotomia lingual na atividade elétrica dos músculos masseter e supra-hióideos e na qualidade da amamentação. Dissertação de Mestrado. Universidade Federal de Pernambuco, Recife, 2019; 107p.

34. SANTOS POM, et al. Frenulotomia lingual em paciente pediátrico: relato de caso. Arch Health Invest, 2018; 7(4):139142.

35. SANTOS S, BARBOSA GF. Reinserção de freio labial superior-relato de caso. Full dentistry in science, 2017; 8(31): 39-42.

36. SILVA VF, et al. Caracterização do frênulo e dos aspectos da língua de indivíduos com esclerose sistêmica. Distúrbios da Comunicação, 2019; 31(1): 95-103.

37. SUZART DD, CARVALHO ARR. Alterações de fala relacionadas às alterações do frênulo lingual em escolares. Revista CEFAC, 2016. 18(6): 1332-1339.

38. VIEIRA KA, MACHADO FG. Frenectomia em odontopediatria: Relato de caso. Revista da AcBO., 2018. 7(2): 23167262.

39. XAVIER MMAPC. Anquiloglossia em pacientes pediátricos. Dissertação (Mestrado em Medicina Dentária). Faculdade de Medicina Dentária, Universidade de Lisboa, Lisboa, 2014; 47p. 